This is the author's version of an article that has been published in the MHI 2019 proceedings.

Changes were made to this version by the publisher prior to publication.

The final version of record is available at https://dx.doi.org/10.1007/978-3-662-59317-2_17

\title{
Potential of Energy Storage Systems for Industrial Robots
}

\author{
Elias Knöchelmann ${ }^{1}$, Jens Kotlarski ${ }^{2}$, Timo Böhm ${ }^{3}$, Svenja Tappe ${ }^{1}$, and \\ Tobias Ortmaier ${ }^{1}$ \\ ${ }^{1}$ Gottfried Wilhelm Leibniz Universität Hannover, Hannover, Germany \\ elias.knoechelmann@imes.uni-hannover.de, \\ 2 forward ttc GmbH, Hannover, Germany \\ jens.kotlaski@forward-ttc.de, \\ 3 Dr. Ing. h.c. F. Porsche AG, Stuttgart, Germany \\ timo.boehm@porsche.de,
}

\begin{abstract}
This paper presents a new approach to estimate the benefit of a energy storage for certain robots. This method can be used directly in the planning phase of production. First, a robot model is developed including the DC grid coupling of the individual drives. This model is validated by several measurements of the absorbed power, brake power and DC grid voltage in a real car body shop. In a next step, the model is used to estimate the potential of an energy storage system for robots in a specific production. The estimation was successfully validated with and without energy storage. In the experimental evaluation an energy saving of $10 \%$ was achieved.
\end{abstract}

Keywords: Industrial Robotics, Energy Efficiency, Energy Storage System, Robot Modeling

\section{Introduction}

The automotive industry is one of the main contributors for advancing automation. The backbone of automation, robots, reached a new sales height in 2017 [11]. The largest part (one third) of these robots are used in the automotive industry [12]. In the past, a lot of work focused decreasing cycle time of robots [10],[4]. Nowadays, through European Regulation [3] and the relationship between production cost and energy consumption, reducing energy consumption of production lines has aroused a significant interest. Robots use up to $8 \%$ of the whole energy needed for production [2]. Hence, a way to decrease production costs is to reduce the energy demand of robots. In recent years, there has been a lot of research in this field [2], which can be classified by the development stages. The sooner energy efficiency is included into the realization of a production line, the more methods are available. For example choosing a robot with a payload of $16 \mathrm{~kg}$ for a task where $10 \mathrm{~kg}$ payload is needed is more energy efficient than choosing a robot with a payload of $200 \mathrm{~kg}$ [2]. These change can only be realized in the planning phase. Optimizing robot trajectory [8], reducing the idle and waiting time by slowing the robots down and change the schedule 
This is the author's version of an article that has been published in the MHI 2019 proceedings.

Changes were made to this version by the publisher prior to publication.

The final version of record is available at https://dx.doi.org/10.1007/978-3-662-59317-2_17
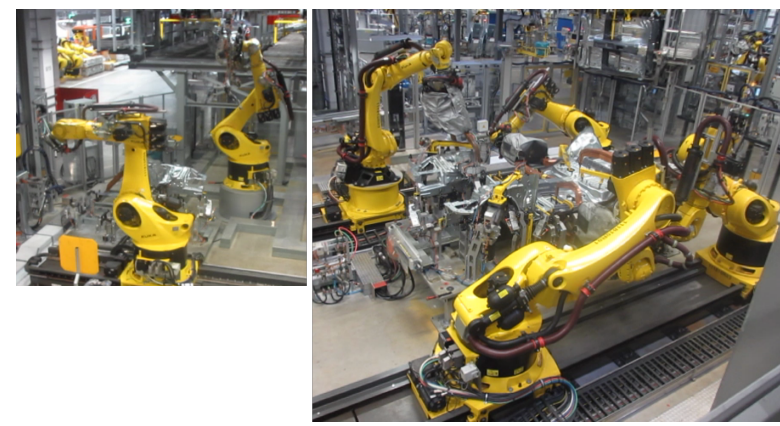

Fig. 1: Picture of the examined production cell.

of robot movement [15] are software-based applications. These methods can be implemented in existing production lines. But, changing trajectories in existing production lines still need manual adjusting: By reducing necessary energy or travel time the robot follows a new path [16], so there might be collusions with other robots or other parts of the production line.

An alternative, which does not change the programming of the robot, is to expand the DC grid capacity [7] [14]:

In production lines, classical robot tasks are transport and positioning. For these applications less precision is needed. Hence they are planed with maximum velocity and acceleration. In deceleration the kinetic energy of the robot converts through servo drives into electrical energy. This happens to all axis at the same time because the robot axis are synchronized to the slowest axis. The regenerative energy is stored in the DC grid capacity until the DC grid voltage hits the critical voltage limit. The brake chopper converts then a specific amount of energy into heat. By adding an energy storage system to the DC grid this conversion can be prevented. This potential savings have been confirmed in production similar application [6], but not real production. Furthermore, there was no guideline which robots needs a energy storage and which does not. The ideal size of the storage system depends on the robot movement and can be estimated by measuring the dissipated chopper energy. However, the measurement of chopper power in active production lines has high safety requirements, as production downtimes lead to high costs.

The aim of this paper is to provide an approach for integrating energy storage systems into production lines. Therefore, a robot model with coupled axis is to be taken into account in the design of the production cell. If it becomes apparent in the design that a robot generates a particularly large amount of braking energy, an energy storage device can be considered directly. In order to implement this procedure, a robot cell with seven robots is investigated. These robots all have different tasks, from welding and gluing to material transport, different weight classes and different numbers of axes. The complete cell is shown in Fig. 1. Measurements are carried out in this cell during operation so that a statement can be made about the necessity of energy storage devices in car body construction cell. 
This is the author's version of an article that has been published in the MHI 2019 proceedings.

Changes were made to this version by the publisher prior to publication.

The final version of record is available at https://dx.doi.org/10.1007/978-3-662-59317-2_17

The paper starts in Section 2 with the modeling of industrial robots. Particular attention is paid to electronic modeling, because this has a great influence on the level of braking energy and thus on the model quality. Section 3 describes the procedure for measuring the chopper power and the absorbed energy. In addition, the model is compared to the measured data. In chapter 4 the robot model is used to estimate the energy savings from an energy storage system. The model results are compared to real measurements. The predicted potential for saving energy is compared with an experimental installation of an energy storage system in Section 5. The paper is closed in Section 6 with a conclusion and an outlook on future work.

\section{Modeling of Industrial Robots}

In earlier publications detailed models for energy prediction have been introduced, e.g. [8] and [15]. Since the main focus of this paper are industrial robots simplifications can be made, like neglecting copper losses in the servo drives. In [16] such a model for energy prediction was introduced. In this paper the main use of the model is chopper power prediction, as it is the main source of energy saving potential. Therefore, changes to existing models have to be made for example adding a DC grid and DC grid capacity.

\subsection{Mechanical Modeling}

This subsection focuses on the mechanical modeling of the robot. The modeling is discreet, since the robot's resonant frequency is far above the time step $\Delta T$ (4 ms) of the model. In most cases, the robot trajectory is defined by the robot motion planning. Hence, the motor torque $\boldsymbol{\tau}(k)$ at the step $k$ can be calculated by

$\boldsymbol{\tau}(k)=\operatorname{diag}\left(\frac{1}{u_{\mathrm{G}, 1}}, \ldots, \frac{1}{u_{\mathrm{G}, n}}\right) \cdot(\boldsymbol{M}(\boldsymbol{q}(k)) \ddot{\boldsymbol{q}}(k)+\boldsymbol{c}(\boldsymbol{q}(k), \dot{\boldsymbol{q}}(k))+\boldsymbol{g}(\boldsymbol{q}(k))+\boldsymbol{h}(\dot{\boldsymbol{q}}(k)))$,

where $\left(u_{\mathrm{G}, 1}, \ldots, u_{\mathrm{G}, n}\right)$ represents the gear factors and $\boldsymbol{q}, \dot{\boldsymbol{q}}, \ddot{\boldsymbol{q}}$ the joint angles, velocities and acceleration given by the motion planning. $\boldsymbol{M}$ contains the moments of inertia, $\boldsymbol{c}$ Coriolis effects and $\boldsymbol{g}$ gravitational effects. The friction model is represented by $\boldsymbol{h}$. The applied friction model is given by,

$$
h_{i}(k)=f_{\mathrm{c}, i} \operatorname{sign}\left(\dot{\varphi}_{i}(k)\right)+f_{\mathrm{v}, i} \dot{\varphi}_{i}(k) .
$$

$f_{\mathrm{c}, i}$ represents the Coulomb friction coefficient for joint $i$ and $f_{\mathrm{v}, i}$ the viscous friction coefficient. For further details on friction see [1]. The motor velocity $\dot{\varphi}_{i}(k)$ can be described by:

$$
\dot{\varphi}_{i}(k)=u_{\mathrm{G}, i} \dot{q}_{i}(k)
$$

Based on this, the mechanical power $P_{\text {mech, } i}(k)$ of the robot can be calculated by:

$$
P_{\text {mech }, i}(k)=\tau_{i}(k) \dot{\varphi}_{i}(k) .
$$

For higher accuracy the counter balancing system can be implemented [13], otherwise the mechanical modeling is completed. 
This is the author's version of an article that has been published in the MHI 2019 proceedings.

Changes were made to this version by the publisher prior to publication.

The final version of record is available at https://dx.doi.org/10.1007/978-3-662-59317-2_17

\subsection{Electrical Modeling}

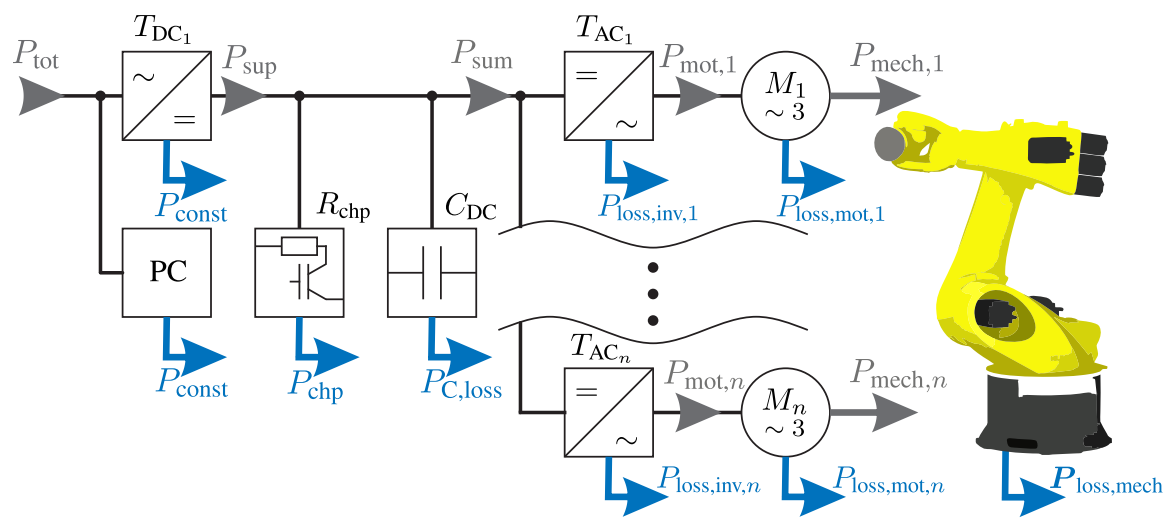

Fig. 2: Detailed energy flow in the investigated robot.

After the mechanical modeling the next step is the electrical modeling. All electrical losses are shown in Fig. 2 and are included in the model. To calculate the motor power $P_{\text {mot, } i}(k)$, a distinction must be made between generator $\left(P_{\text {mech }, i}(k)<0\right)$ :

$$
P_{\mathrm{mot}, i}(k)=P_{\mathrm{mech}, i}(k) \cdot \eta,
$$

and motor $\left(P_{\text {mech }, i}(k) \geq 0\right)$ operation mode:

$$
P_{\mathrm{mot}, i}(k)=P_{\mathrm{mech}, i}(k) \cdot \frac{1}{\eta}
$$

where $\eta$ is the motor efficiency. The inverter power then results to

$$
P_{\mathrm{inv}, i}(k)=P_{\mathrm{mot}, i}(k)+P_{\mathrm{loss}, \mathrm{inv}, i}(k),
$$

with $P_{\text {loss,inv }}(k)$ as the constant inverter losses. To calculate the changes the DC grid Power $P_{\text {sum }}$ has on the DC grid voltage $U_{\mathrm{dc}}$, the energy $\Delta W_{\text {sum }}(k)$ of a specific time step $\Delta T$ needs to be calculated:

$$
\Delta W_{\text {sum }}(k)=P_{\text {sum }} \Delta T=\left(\sum_{i=1}^{n} P_{\text {inv }, i}(k)\right) \Delta T ; \Delta T=t(k)-t(k-1) .
$$

The number $n$ of motors depends on the chosen robot. A prediction for the intermediate circuit voltage $U_{\text {predict }}(k)$ in current step $k$ can be made via the field energy in the capacitor. It is assumed that the energy stored in the field corresponds to the energy sum $\Delta W_{\text {sum }}(k)$. The basic idea comes from

$$
U_{\text {predict }}(k)=\sqrt{\frac{2}{C_{\mathrm{dc}}} \cdot\left|\Delta W_{\mathrm{sum}}(k)\right|+U_{\mathrm{dc}}^{2}(k-1)}-U_{\mathrm{C}, \text { loss }},
$$


This is the author's version of an article that has been published in the MHI 2019 proceedings.

Changes were made to this version by the publisher prior to publication.

The final version of record is available at https://dx.doi.org/10.1007/978-3-662-59317-2_17

where $C_{\mathrm{dc}}$ is the DC grid capacitance, $U_{\mathrm{dc}}(k-1)$ is the DC grid voltage from the previous step, and $U_{\mathrm{C}, \text { loss }}$ is the losses caused by the leakage currents in the capacitor. The charging and discharging process and thus the current DC grid voltage $U_{\mathrm{dc}}$ of the capacitor is calculated as follows:

$$
U_{\mathrm{dc}}=U_{\text {predict }}(k)+\left(U_{\text {predict }}(k)-U_{\mathrm{dc}}(k-1)\right) \mathrm{e}^{-\frac{\Delta T}{R_{\mathrm{c}} C_{\mathrm{dc}}}},
$$

with $R_{\mathrm{C}}$ as internal resistance of the DC grid capacitance. Now based on the DC grid voltage a distinction is made between three cases:

1. Voltage is too low

2. Voltage is too high

3. Voltage is in given boundaries

If the $\mathrm{DC}$ grid voltage $U_{\mathrm{dc}}$ is below the minimum value, it is set to this value; if it is above the maximum value, it is also set to the maximum value. The difference between the predicted and current DC grid voltage $\Delta U_{\mathrm{dc}}(k)$ is then calculated.

$$
\Delta U_{\mathrm{dc}}(k)=U_{\text {predict }}(k)-U_{\mathrm{dc}}(k) .
$$

Based on this difference, it is decided whether the DC grid is charged by the grid or the excess energy is converted into heat to protect the DC grid. Is $\Delta U_{\mathrm{dc}}(k) \leq 0$, then the chopper power $P_{\mathrm{chp}}(k)$ is set to zero in this step and the power $P_{\text {sup }}(k)$ with which the DC grid is loaded is calculated as:

$$
P_{\text {sup }}(k)=\frac{\Delta W_{\text {sup }}(k)}{\Delta t} ; \Delta W_{\text {sup }}(k)=-\frac{1}{2} \cdot C_{\mathrm{dc}} \cdot\left(U_{\text {predict }}^{2}(k)-U_{\mathrm{dc}}^{2}(k)\right) .
$$

Is $\Delta U_{\mathrm{dc}}(k)>0$, then the supply power $P_{\text {sup }}(k)$ is set to zero and chopper power $P_{\text {chp }}(k)$ is calculated as:

$$
P_{\text {chp }}(k)=\frac{\Delta W_{\text {chp }}(k)}{\Delta t} ; \Delta W_{\text {chp }}(k)=\frac{1}{2} \cdot C_{\text {dc }} \cdot\left(U_{\text {predict }}^{2}(k)-U_{\mathrm{dc}}^{2}(k)\right) .
$$

The total energy absorbed by the grid $E_{\text {tot }}$ is calculated as follows:

$$
E_{\mathrm{tot}}=\left(\sum_{k=1}^{n_{\mathrm{t}}} P_{\mathrm{tot}}(k)\right) \Delta T ; \quad P_{\mathrm{tot}}(k)=P_{\text {sup }}(k)+P_{\text {const }},
$$

with $P_{\text {const }}$ as constant losses from the robot controller and $n_{\mathrm{t}}$ as the number of time steps. In [9], a similar approach is used, except that the entire energy is always fed into the DC grid capacity and, if necessary, readjusted by the grid. Here, only a part is stored in the DC grid capacity. The rest always comes from the grid or is converted into heat.

\section{Production Line}

As a model-based estimation of potential energy savings is targeted in tis paper, a validation of the robot model must be performed first. The measurements are carried out at a car body construction cell with seven industrial robots. The robots have payloads ranging from 200 to $500 \mathrm{~kg}$ and their applications are gluing, welding and part transfers. The number of joints range between six and seven (see Table 1). The entire cell with all seven robots is displayed in Fig. 1. 
This is the author's version of an article that has been published in the MHI 2019 proceedings.

Changes were made to this version by the publisher prior to publication.

The final version of record is available at https://dx.doi.org/10.1007/978-3-662-59317-2_17

\subsection{Measurement Setup}

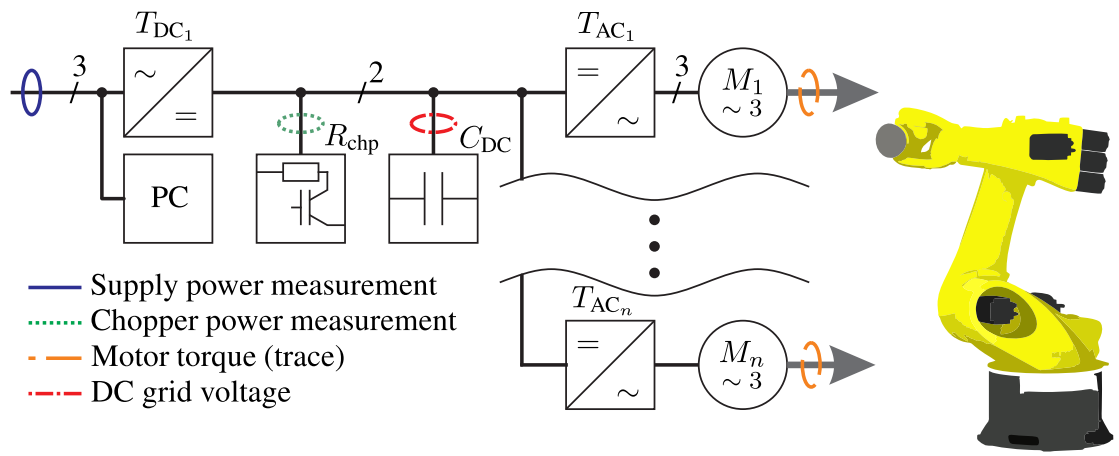

Fig. 3: Schematic representation of the measuring points.

As previously said, an energy storage system prevents that DC grid energy gets converted into heat. Therefore, the amount of copper energy needs to be measured as well as the total used power of the robot for validation of the energy model. For the purpose of long-term measurement without effecting the material flow of the production line, a special measuring box form developed by the forward ttc $\mathrm{GmbH}$ is used. It allows for an accurate measurment of chopper power emitting pulses with an average duration of $0.5 \mathrm{~ms}$ over $2 \mathrm{~h}$. In addition, the DC grid voltage and motor torques for identification of the inverse dynamic parameters are traced using the robot controller. In Fig. 3 the measuring points are displayed.

In Fig. 4 the measurement of one robot is displayed. The upper plot shows the total power consumed by the robot in blue and the braking power in red. The lower plot shows the energy consumed by the robot in blue and the corresponding braking energy in red. $P_{\text {chopper }}$ as well as $E_{\text {chopper }}$ result from the sum of both chopper resistors of the robot.

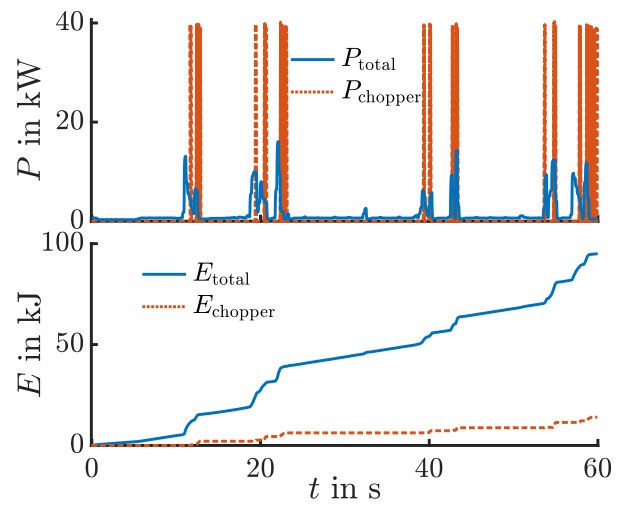

Fig. 4: Measurement of the total and chopper power/energy of a robot. 
This is the author's version of an article that has been published in the MHI 2019 proceedings.

Changes were made to this version by the publisher prior to publication.

The final version of record is available at https://dx.doi.org/10.1007/978-3-662-59317-2_17

\subsection{Model Validation}

The recorded measurements are used to validate the model. The traced motor torque can be used for identification. The focus of this paper is on the electrical equivalent circuit model. Therefore, the traced motor torques are used as model input for a better evaluation of the validation in the course. The simplified energy consumption model with identified dynamic parameters has already been validated in [16] and has a remaining model deviation of approx. $5 \%$ over the whole spectrum of operating temperatures [5].
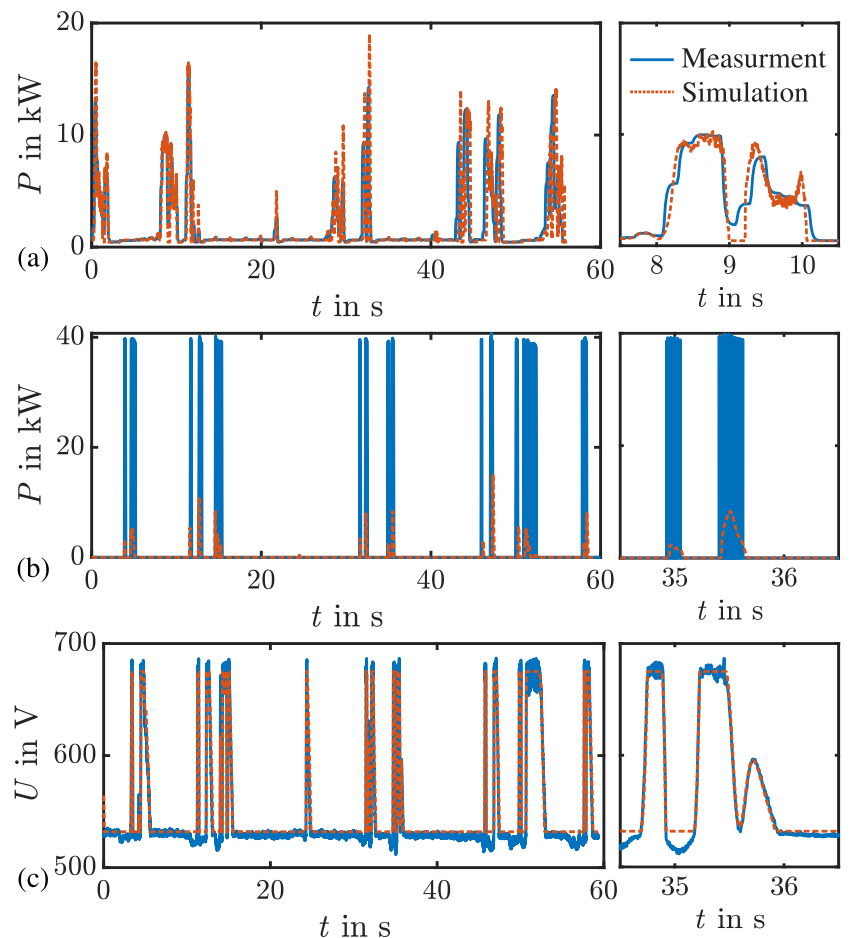

Fig. 5: Comparison of simulation and measurement of total power (a), chopper power (b) and DC grid voltage (c).

The comparison between model and measurement is shown in Fig. 5. The power consumption, Fig. 5.a, between simulation and measurement is well matched. The energy error is up to $3 \%$ for all seven robots only.

The model deviation in the zoom comes from the measuring principle of the power meter. Fast power changes are not transmitted directly by the measuring transformer. However, the absorbed energy is very accurately measured. In the brake power measurement, Fig. 5.b, the energy error is up to $8 \%$. The visible difference comes from the fact that brake power is emitted in 0.5 ms pulses over two $21 \Omega$ resistors in the experimental setup. The simulated chopper power is lower because the chopper power generated in each time step (4 ms) of the 
This is the author's version of an article that has been published in the MHI 2019 proceedings.

Changes were made to this version by the publisher prior to publication.

The final version of record is available at https://dx.doi.org/10.1007/978-3-662-59317-2_17

model is delivered constantly over the whole time step. This also has an effect on the voltage, since chopper starts at a fixed voltage and the voltage of the real robot fluctuates due to the chopper pulses. This can be seen from the zoom in Fig. 5.c. In the simulation, the motor power increase cannot reduce the DC grid voltage further than a certain limit value. However, the measured curve in Fig. 5.c shows that the voltage also falls below this limit. Nevertheless, the voltage flanks, which determine the status of the model (Section 2.2), fit together very well. Therefore, it can be summarized, that the model reproduces all relevant aspects with sufficient accuracy.

\section{Model-based Analysis of Potential Energy Savings}

In order to demonstrate the possible saving potential by installing an energy storage device, measurements are performed in the robot cell outlined in Section 3.1. In addition, the traced data is used to make an extrapolation using the energy model. The results are depicted in Table 1 . To evaluate the results, they are extrapolated to one year using a fictitious production example. It is assumed that the robots work five days a week in three-shift operation, i.e. 24 hours a day. Based on 50 working weeks per year, this results in 250 days. It is noticeable that the first robot has the greatest brake losses, which is also supported by the low DC grid capacity, indicating, that only a small amount of energy can be saved in the DC grid.

The simulation results can be additionally compared to the previously measured dissipated energy amount. Results show again, that the model can mirror the actual robot behavior with good accuracy.

Table 1: Comparison of the savings potential by installing an energy storage system

\begin{tabular}{c||c|c|c|c|c|c|c} 
robot & 1 & 2 & 3 & 4 & 5 & 6 & 7 \\
\hline \hline joints & 6 & 7 & 7 & 7 & 7 & 7 & 6 \\
\hline DC grid capacity in $\mu \mathrm{F}$ & 1060 & 1790 & 1790 & 1790 & 1790 & 1172 & 1060 \\
Simulation \\
chopper Energy p.a. in kWh & 2609 & 1707 & 839 & 1282 & 1310 & 1552 & 1379 \\
\hline savings potential p.a. in \% & 15 & 12 & 9 & 9 & 10 & 12 & 14 \\
Measurement & \\
chopper Energy p.a. in kWh & 2821 & 1770 & 890 & 1373 & 1408 & 1632 & 1528 \\
\hline savings potential p.a. in \% & 17 & 12 & 9 & 10 & 11 & 11 & 15
\end{tabular}

\section{Experimental Evaluation of Energy Savings}

In addition to the previews calculated potential energy savings, this chapter deals with the actual savings achieved, when installing an energy storage device into 
This is the author's version of an article that has been published in the MHI 2019 proceedings. Changes were made to this version by the publisher prior to publication.

The final version of record is available at https://dx.doi.org/10.1007/978-3-662-59317-2_17

the exemplary robot cell. To install the energy store, the relevant robot must be switched off. Exemplary robot seven was used to install the energy storage. The energy storage device used has a fixed energy capacity of $1600 \mathrm{~J}$.

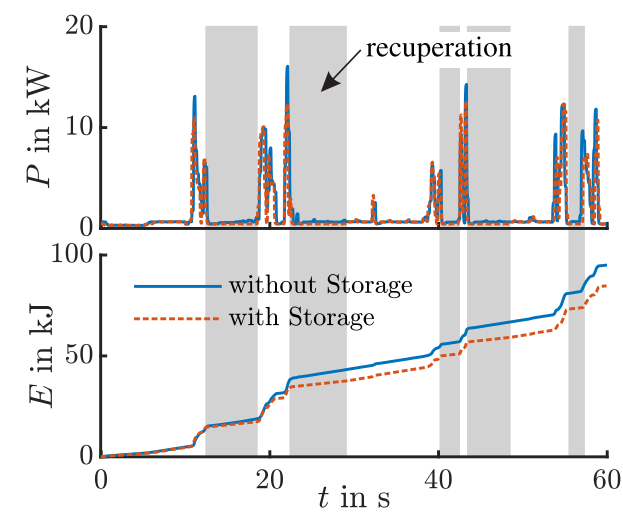

Fig. 6: Comparison of power consumption with and without energy storage system.

The power consumed before and after installation is compared with each other in Fig. 6. The power curves with the energy storage system (red) and without the energy storage system (blue) are shown in the upper half, the corresponding energy curves in the lower half. The areas in which the energy from the energy storage device is pushed back into the robot are highlighted in gray.

The savings resulting from the installation of the energy storage device are $10.8 \%$ and $1017 \mathrm{kWh}$, see Table 2 . The model-based approach estimated savings of $14 \%$ ( $\hat{=} 1379 \mathrm{kWh}$ ), see Table 1 . This difference is due to the fact that the efficiency of the energy storage is not taken into account in the potential calculation. It is also assumed that the energy accumulator can absorb the entire braking energy.

The energy capacity of the energy storage device used for the experiment is a little too small, because $641 \mathrm{~J}$ are dissipated every robot cycle (p.c.) even with the energy storage system. Increasing the storage capacity will help to reduce the remaining dissipations, unfortunately, the savings will then be matched by larger investments.

Table 2: Comparison of the savings potential by installing an energy storage system

\begin{tabular}{c|c|c|c|c} 
Robot & $\begin{array}{c}\text { used energy p.c. } \\
\text { without E. storage }\end{array}$ & $\begin{array}{c}\text { used energy p.c. } \\
\text { with E. storage }\end{array}$ & $\begin{array}{c}\text { savings p.a. } \\
\text { in } \mathrm{kWh}\end{array}$ & $\begin{array}{c}\text { savings p.a. } \\
\text { in \% }\end{array}$ \\
\hline \hline 7 & $95033 \mathrm{~J}$ & $84747 \mathrm{~J}$ & 1017 & 10.8
\end{tabular}


This is the author's version of an article that has been published in the MHI 2019 proceedings.

Changes were made to this version by the publisher prior to publication.

The final version of record is available at https://dx.doi.org/10.1007/978-3-662-59317-2_17

\section{Conclusion}

In this paper, a model-based approach for analyzing energy savings through added storage system was presented. It is based on an accurate model of the dissipated energy. This modeling was successfully validated by measurements of a robot cell from a car body shop. The validated simulation results show great potential for the installation of energy storage devices. This potential has been confirmed by a measurement with and without energy storage. Measurements underlined the previous analysis of potential savings. Despite that the used storage system was slightly too small and its losses were not included in robot model, a total savings of $10.8 \%$ (compared to the $14 \%$ in simulation) were achieved.

In the near future, the model can be expanded to include an energy storage unit. With this extension it will be possible to take the discrete sizes of commercial energy storages and its losses directly into account.

\section{References}

1. B. Armstrong-Hélouvry, P. Dupont, and C. C. de Wit. A survey of models, analysis tools and compensation methods for the control of machines with friction. Automatica, 30(7), 1994.

2. M. Brossog, M. Bornschlegl, J. Franke, et al. Reducing the energy consumption of industrial robots in manufacturing systems. The International Journal of Advanced Manufacturing Technology, 78(5-8), 2015.

3. E. Commission. 2030 energy strategy.

4. O. Dahl and L. Nielsen. Torque-limited path following by online trajectory time scaling. IEEE Transactions on Robotics and Automation, 6(5), 1990.

5. K. Eggers, E. Knöchelmann, S. Tappe, and T. Ortmaier. Modeling and experimental validation of the influence of robot temperature on its energy consumption. 2018.

6. O. H. für angewandte Wissenschaften. Mit energiespeichern energie dynamisch sparen, 2016.

7. C. Hansen, K. Eggers, J. Kotlarski, and T. Ortmaier. Comparative evaluation of energy storage application in multi-axis servo systems. Proceedings of The 14th IFToMM World Congress, 2015.

8. C. Hansen, J. Kotlarski, and T. Ortmaier. Experimental validation of advanced minimum energy robot trajectory optimization. In Advanced Robotics (ICAR), 2013 16th International Conference on, 2013.

9. C. Hansen, J. Kotlarski, and T. Ortmaier. Optimal motion planning for energy efficient multi-axis applications. Mechatronics and Automation, 4(3), 2014.

10. J. M. Hollerbach and G. Sahar. Planning of minimum-time trajectories for robot arms. 1986

11. IFR. Ifr ceo round table: 20 june 2018 munich, 2018.

12. G. Litzenberger. Welcome to ifr eb meeting in carate brianza. 2016.

13. D. Meike, M. Pellicciari, and G. Berselli. Energy efficient use of multirobot production lines in the automotive industry: Detailed system modeling and optimization. Automation Science and Engineering, IEEE Transactions on, 11(3), 2014.

14. D. Meike and L. Ribickis. Recuperated energy savings potential and approaches in industrial robotics. In Automation Science and Engineering (CASE), 2011.

15. M. Pellicciari, G. Berselli, F. Leali, and A. Vergnano. A method for reducing the energy consumption of pick-and-place industrial robots. Mechatronics, 23(3), 2013.

16. Z. Ziaukas, K. Eggers, J. Kotlarski, and T. Ortmaier. Optimizing ptp motions of industrial robots through addition of via-points. In Proceedings of the 14th I. Conference on Informatics in Control, Automation and Robotics, 2017. 